\title{
CRECIMIENTO Y SUPERVIVENCIA EMPRESARIAL EN EL SECTOR DE FABRICACIÓN DE CALZADO EN ECUADOR
}

Lilián Victoria Morales-Carrasco

Universidad Técnica de Ambato Ecuador

Karla Estefanía Morales-Ramos Universidad Técnica de Ambato Ecuador

Alexandra Tatiana Valle-Álvarez Universidad Técnica de Ambato Ecuador 
Panorama Económico, Vol. 25 - No. 1 (Enero - Marzo de 2017), pp. 43-56

Lilián Victoria Morales-Carrasco

Karla Estefanía Morales-Ramos

Alexandra Tatiana Valle-Álvarez

\title{
Crecimiento y supervivencia empresarial en el sector de fabricación de calzado en Ecuador
}

\begin{abstract}
Resumen
El presente estudio fue motivado por casos de éxito de empresas dedicadas a la fabricación de calzado en Ecuador, en particular en la provincia de Tungurahua, y se explicaron los determinantes de la capacidad de supervivencia de estas empresas establecidas en el mercado ecuatoriano a lo largo de los años. Los datos empleados para el analisis corresponden a indicadores producción, ventas y capacidad instalada medidos a partir de las cuentas de los estados financieros de las empresas. Por otro lado, la supervivencia considera indicadores edad y tamaño inicial de las empresas, Mediante un análisis estadístico de correlación, regresión lineal múltiple y anova, se evidencia una alta y positiva correlación entre las ventas y producción empresarial con la supervivencia de las firmas para los casos estudiados.

Palabras clave: crecimiento empresarial, supervivencia de empresas, sector fabricación de calzado.

Clasificación JEL: D92, D21, L67
\end{abstract}

\section{Growth and survival of firms in the footwear manufacturing sector in Equador}

\begin{abstract}
The present study was motivated by successful footwear manufacturing sector in Equador, particularly in Tungurahua region, and explains the determinants of the survival characteristics of these companies established in the Ecuadorian market. Data used for the quantitative analysis corresponds to production, sales and installed capacity, obtained from the firms' financial statements; On the other hand, survival characteristics consider age and initial size of firms. A statistical analysis of correlation, multiple linear regression and anova has been applied. The main results show a high positive correlation between firm's sales and production with firm's survival.
\end{abstract}

Keywords: business growth, firm survival, footwear manufacturing sector.

JEL Classification: D92, D21, L67

\section{La croissance et la survie des entreprises manufacturières du secteur des chaussures en Équateur}

\section{Résumé}

La présente étude est motivée par les succès d'entreprises de fabrication de chaussures dans la province de Tungurahua en Équateur. Les déterminants de la capacité de survie de ces sociétés établies sur le marché équatorien au fil des ans ont été expliqués. Pour la variable de croissance indépendante, les informations utilisées correspondent aux indicateurs production, ventes et capacité installée mesurés avec les comptes des états financiers pour la période 2000-2012. Pour la variable de survie dépendante, les indicateurs d'âge et de taille initiale des entreprises ont été utilisés, qui ont été établis à l'aide d'une enquête. Une analyse statistique de la corrélation, de la régression linéaire multiple et de l'anova a été appliquée. Les principaux résultats montrent une corrélation forte et positive entre les variables ventes et production avec survie, ce qui suggère que lorsque les entreprises augmentent leur production et leurs ventes, la probabilité de survie augmente dans les cas étudiés.

Mots-clés: Croissance des entreprises, survie des entreprises, secteur de la fabrication de chaussures. Nomenclature JEL: D92, D21, L67 


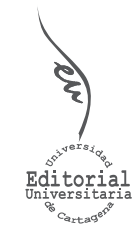

Artículo de Investigación

\section{Crecimiento y supervivencia empresarial en el sector de fabricación de calzado en Ecuador}

INFORMACIÓN DEL ARTÍCULO

Recepción de artículo: 18/10/2016

Concepto de evaluación: 10/11/2016

Aceptación de artículo: 15/12/2016
Lilián Victoria Morales-Carrasco* Universidad Técnica de Ambato, Ecuador

Karla Estefanía Morales-Ramos Universidad Técnica de Ambato, Ecuador

Alexandra Tatiana Valle-Álvarez Universidad Técnica de Ambato, Ecuador

\section{INTRODUCCIÓN}

El sector manufactura está considerado como uno de los más importantes dentro de los aportantes al crecimiento de la economía en el Ecuador (Plan Nacional del Buen Vivir 2013-2017), pues contribuyó en el año 2011, con un 12,8\% al PIB real y un 14,78\% a la generación de empleo de la concentración de mano de obra nacional, empleando hasta 100 mil trabajadores (Instituto Nacional de Estadísticas y Censos, 2013). Entre el 2012 y el 2014, los ingresos anuales en la provincia de Tungurahua, bordearon los USD 139 millones (Ministerio de Industrias y Productividad, 2014), debido al aumento de la manufacturación. En el Ecuador existen 870 establecimientos que se dedican a la producción de calzado según datos del Censo Económico 2010 (Encuesta de Manufactura y Minería-INEC, 2010). Por lo expuesto, el sector de fabricación de calzado ha sido motivo de estudio en la presente investigación, cuyo objetivo fue analizar los determinantes de la supervivencia empresarial en casos de éxito basados en la hipótesis de que el crecimiento está relacionado con la supervivencia (permanencia en el mercado) de las empresas y además incidiendo positivamente en ella.

A continuación se presenta la revisión de la literatura, con respecto al crecimiento empresarial y la supervivencia de las empresas, en donde se establecen los factores determinantes, así como los indicadores analizados desde distintas perspectivas por varios autores.

\footnotetext{
* Autor para correspondencia

Correos electrónicos: lilianmorales@uta.edu.ec*, karlamorales3181@uta.edu.ec, alexandratvalle@uta.edu.ec
} 


\subsection{REVISIÓN DE LA LITERATURA}

El crecimiento empresarial es un "proceso de adaptación a los cambios exigidos por el entorno o promovidos por el espíritu emprendedor del directivo" por lo que la empresa se verá obligada a desarrollar o ampliar su capacidad productiva mediante el ajuste o adquisición de nuevos recursos (Blázquez, Dorta, y Verona, 2006) o al "manejo adecuado de recursos que poseen las organizaciones al interior, es decir, a la acción compleja de las modificaciones internas que una organización pueda tener y que desembocan en cambios de las características que identifican a una empresa" (Muñoz-Salgado, Nava-Rogel y Rangel, 2013).

Si se desea impulsar a la empresa a un crecimiento dentro de un tiempo establecido, es necesario implantar estrategias de crecimiento basadas en la exclusividad y complementariedad de los conocimientos estratégicos, creación de nuevo conocimiento, generación de valor y apropiación de rentas derivadas de los mismos de forma inmediata y duradera a través del tiempo (Guadamillas, 2006).

Por otro lado, las estrategias de internacionalización son un medio de crecimiento empresarial, pues se debe mejorar la productividad e innovarla, es así que consigue una condición que le garantice el éxito y la estabilidad laboral (Puerto, 2010).

Otros autores afirman que el conocimiento, las ideas, la creatividad, los mejores recursos, el talento humano y las herramientas del capital intelectual deben ser un elemento clave para la expansión de la empresa, donde la comunicación juega el rol más determinante y el trabajo en equipo el motor impulsor de un gran logro a futuro evidenciando así diferencias en la calidad, efectividad, servicio y posicionamiento, que aceleran el proceso de crecimiento empresarial, por lo que se debería considerar a la gestión del conocimiento como pieza fundamental para el crecimiento empresarial llegando así a formar parte de un activo más de la empresa (Velásquez, Quintero, Sánchez y Burgos, et.al., 2010).

Otras investigaciones apoyan el hecho de que si se toma a la responsabilidad social empresarial de manera global para una organización se está incentivando a un crecimiento empresarial por medio de la transmisión de valor agregado a la sociedad que a futuro se transformará en una ventaja netamente competitiva, por lo que trabajan conjuntamente con la responsabilidad económica, es decir, ofertar al consumidor bienes y servicios a precios accesibles que cumplan con las necesidades del consumidor, la responsabilidad legal basada en cumplimiento de las leyes y normas que rigen a la entidad ya sean estas nacionales o internacionales con el fin de dar confiabilidad a los clientes y estabilidad a la empresa y la responsabilidad voluntaria que viene siendo el valor agregado de la sociedad para con la empresa, de esta manera se afirma que la responsabilidad social dentro de los planes estratégicos de la empresa aumenta la rentabilidad (Aguilera y Puerto, 2012), esta última podría ser el indicador del crecimiento de una empresa.

El crecimiento empresarial proviene de diferentes aspectos generales entorno a la empresa y la aplicación de las estrategias se ve limitada por el cumplimiento de un conjunto de indicadores como el tamaño, la edad, el número de empleados, entre otros, los cuales determinan cuándo es más eficaz considerar una estrategia de crecimiento (Aguilera y Virgen, 2014). 
Finalmente, es relevante para el progreso de una organización en un ambiente determinado, encaminar sus acciones al cumplimiento de sus objetivos; definir su capacidad interna y dar respuesta a las exigencias del entorno (Aguilera, 2010).

El término supervivencia se refiere a la permanencia de las empresas en el mercado, es decir, determinar el grado de estabilidad laboral que ofrece la compañía así como el porcentaje de riesgo que esta presenta. En este apartado se analizan estudios que aclaran este panorama desde diferentes investigaciones.

Es así que "desde la creación de la empresa los emprendedores inician con la conservación del negocio, haciendo todo lo posible para que se adapte al entorno competitivo de la mejor forma posible" llamándolo supervivencia empresarial (Arias y Quiroga, 2008); adicionalmente cuanto más grande es el tamaño de la organización será mayor la probabilidad de seguir en el mercado (Bordonaba, Lucía y Polo, 2008).

En otros estudios se afirma que el tamaño, la pertenencia, el número de años desde su iniciación y el sector son importantes a la hora de definir cuáles son los factores que condicionan el éxito de la supervivencia, información diagnóstica que sirve como instrumento para preseleccionar a las empresas que necesiten de una atención especializada, en empresas que han optado por el crecimiento como estrategia para perdurar en el tiempo. De tal forma que exista una gestión más eficiente del tiempo dedicado por el experto, analista financiero o la autoridad supervisora, para conseguir una igualdad en los juicios de valor emitidos (Segovia y Camacho, 2012).

Para poder hablar de permanencia en el mercado es necesario tener claro el objetivo que persiguen las empresas según su estructura de propiedad del capital, y sus metas a alcanzar, además de identificar el vínculo sea socio o trabajador con la empresa y un esfuerzo más intenso en el trabajo, lo que incide positivamente en un aumento de la productividad de los trabajadores, además que de esta manera se estaría asegurando el trabajo estable y la continuidad de sus operaciones, es decir asegurando una estabilidad laboral (Melgarejo, Arcelus y Simón, 2007).

La literatura indica que la inversión en investigación y desarrollo (I+D) es la base sólida de la sociedad del conocimiento, ayudando significativamente al desarrollo económico y el progreso del bienestar social. En algunos estudios se encuentra que la rentabilidad, garantías a terceros, la eficiencia y la edad de las empresas afectan de manera negativa en la probabilidad de fracaso empresarial. Es de suma importancia que al momento de innovar la variable I+D sea rentable, con el fin de generar recursos suficientes para la financiación de la misma y la continuidad de la empresa (Baltar, Fernández, Vivel, Neira, 2011).

Desde otra perspectiva, son dos los factores esenciales influyentes en la supervivencia de las empresas ya sean estos el emprendimiento social y el emprendimiento de negocios; el primero es consecuencia de la motivación para emprender por parte de la empresa y el segundo busca cumplir con la misión de la empresa, identificar el tipo de oportunidades al iniciar la empresa así como las dificultades que se presenten, las restricciones de sus recursos y la medición de los resultados; aunque si se analiza desde un punto de vista general, ambos constituyen actividades innovadoras que identifican las oportunidades parecidamente y enfrentan retos en las 
mismas circunstancias por lo que les hace relacionarse entre sí, por lo que en general, la formación, experiencia y motivación del empresario junto con el sector y el tamaño de la empresa vienen siendo los factores que juegan a favor de la supervivencia de la empresa (Simón, 2012).

El crecimiento empresarial y la supervivencia de las empresas, son variables que juegan un rol muy importante en la economía, pues en base a estas se verá reflejado el progreso continuo de las empresas y el sector económico al que pertenecen.

Hoy en día el conocimiento juega un rol importante en cuanto al desarrollo tanto social como económico. Algunos trabajos de investigación determinan que la creación, distribución y uso del conocimiento impulsan directamente el crecimiento económico, la riqueza y el empleo, hablando así en términos de crecimiento de las ventas, la rentabilidad o la creación de empleo. Se sustenta la existencia de un efecto positivo de la I+D en el crecimiento y la tasa de supervivenciadelaindustria manufacturera que tienen un mayor gasto en I+D y los efectos sobre el crecimiento de las ventas netas diferenciando el componente sectorial en función de su nivel tecnológico y de la intensidad de conocimiento aplicada (García y Romero, 2010).

Después de analizar varios estudios sobre el crecimiento empresarial y la supervivencia, podemos dar por entendido que ambos tienen una relación directa, pues existen variedad de factores que direccionan a las empresas a avanzar continuamente, destacando la actitud emprendedora del empresario para acoger dichos recursos y ponerlos en práctica, así como factores externos netamente dependientes del entorno en el que se desenvuelve la empresa, de tal forma que al pasar los años, las empresas cuentan con varias estrategias que les permite gozar de continuidad y estabilidad en el mercado, si se las emplea correctamente.

En el presente artículo se establece la relación estadística existente entre la variable crecimiento y la variable supervivencia de las empresas del sector fabricación de calzado (CIIU C1520.01) de la provincia de Tungurahua en Ecuador, así como la incidencia del crecimiento en la supervivencia.

\section{DATOS Y METODOLOGÍA}

\subsection{Crecimiento Empresarial}

Para medir el crecimiento empresarial (variable independiente) se realizó una recopilación documental de la base de datos dela Superintendencia de Compañías para obtener información acerca de los Estados Financieros de las empresas de fabricación de calzado de la provincia de Tungurahua bajo el control y vigilancia de la Superintendencia de Compañías (Superintendencia de Compañías, 2013-2017). Se trabaja con las cuentas activo total, costo de ventas e ingresos operacionales, debido a la significancia de éstas, en el estado de situación financiera y en los resultados de las empresas durante el período 2000 - 2012. En coherencia con la literatura revisada, existen distintos tipos de indicadores y factores del crecimiento empresarial, las cuentas mencionadas permitieron medir el crecimiento en capacidad instalada, producción y ventas respectivamente (Véase tabla 1) para las cuatro empresas seleccionadas en el muestreo por criterio, de una población a nivel nacional de veinte compañías que cumplían con información de estados financieros continuos para el período establecido, es decir, trece años de información para cada compañía. 
Tabla 1: Indicadores de Crecimiento Empresarial

\begin{tabular}{|c|c|}
\hline INDICADORES & CUENTAS CONTABLES \\
\hline $\begin{array}{c}\text { Capacidad } \\
\text { Instalada }\end{array}$ & $\begin{array}{c}\text { Activo Total } \\
(2000 / 2012)\end{array}$ \\
\hline Producción & $\begin{array}{c}\text { Costo de Ventas } \\
(2000 / 2012)\end{array}$ \\
\hline Ventas & $\begin{array}{c}\text { Ingresos Operacionales } \\
(2000 / 2012)\end{array}$ \\
\hline
\end{tabular}

Elaboración propia

Fuente: Superintendencia de Compañías

Cabe indicar que se tomaron 13 años de información financiera debido a que la Superintendencia de Compañías tiene publicados los Estados Financieros desde el año 2000 siendo el mayor rango que se pudo tomar hasta el 2012, debido a que el estudio de supervivencia permite observar el recorrido en el tiempo y el comportamiento de las principales cuentas. Por otro lado, una empresa que supera los primeros tres años de existencia presenta probabilidad de permanecer en el mercado, es decir, de sobrevivir y aquellas que han cumplido cinco años podrían considerarse empresas establecidas. En el caso del presente estudio, los años subsiguientes, al umbral de los primeros tres años, fueron de primordial importancia para el análisis de crecimiento y su posterior incidencia en la supervivencia.

Los datos proporcionados por las cuentas de los Estados Financieros, permitieron realizar en primer lugar un análisis descriptivo de la evolución del crecimiento por cada empresa y posteriormente se introdujeron los promedios en el software estadístico $R$ Project para la comprobación de la hipótesis.

\subsection{Supervivencia Empresarial}

A fin de obtener información acerca de la variable supervivencia (permanencia en el mercado) de las empresas se aplicó una encuesta y se procedió de la misma manera que para el casodela variablecrecimiento,es decir, la encuesta fue dirigida a cuatro de las veinte empresas de fabricación de calzado a nivel nacional, que se encuentran bajo el control y vigilancia de la Superintendencia de Compañías del Ecuador y que fueron tomadas en base al muestreo por criterio al cumplir con los 13 años de información de estados financieros. Estas empresas además funcionan en la provincia de Tungurahua. Se averiguó acerca de la edad medida en número de años de la empresa desde el inicio de actividades $^{1}$ y el tamaño inicial medido en número de empleados.

Para determinar la edad de las empresas se aplicó la fórmula:

\section{[1] (Año actual - Año de Inicio) - 1}

donde año actual es el año hasta donde se dispone de Estados Financieros de la Superintendencia de Compañías, es decir hasta el año 2012, el año de inicio es el año en el que la empresa comenzó con sus actividades y -1 si la empresa inició sus actividades a finales de año, para evitar un sesgo en la información.

Al igual que en el caso de la variable crecimiento, también para el estudio de la variable supervivencia los datos permitieron un análisis descriptivo por un lado y posteriormente se utilizaron

$1 \mathrm{El}$ inicio de actividades no tiene por qué coincidir con el año de registro en la Superintendencia de Compañías, ya que algunas inician como empresas de hecho y posteriormente toman la decisión estratégica de constituirse legalmente en compañías. Esta pregunta en el cuestionario permitió detectar la verdadera edad de las empresas o inicio de actividades. 
para la comprobación estadística de la hipótesis, al ingresar los datos en el software estadístico $R$ Proyect.

\subsection{Variación del crecimiento empresarial}

Para determinar los niveles de crecimiento empresarial a través de las cuentas seleccionadas Costo de Ventas, Activo Total e Ingresos operacionales, provenientes de los Estados Financieros, se utilizó la fórmula de la variación, a fin de obtener el porcentaje anual de variación de cada indicador. Posteriormente, se utiliza un promedio de la variación para el análisis estadístico.

\subsection{Modelo estadístico}

Después de la revisión de la literatura, acerca del crecimiento y la supervivencia de las empresas se plantea la siguiente hipótesis:

\section{H1:}

El crecimiento empresarial incide en la supervivencia (permanencia en el mercado) de las empresas del sector de fabricación de calzado.

La hipótesis inicial se plantea en el modelo estadístico:

[2] Supervivencia $=f\left\{\beta_{0}+\beta_{1}\right.$ capacidadins $+\beta_{2}$ produccion $+\beta_{3}$ ingresosop $\left.+e\right\}$

Numéricamente se puede cuantificar el grado de relación lineal mediante la matriz de coeficientes de correlación. Seguidamente se realizó el cálculo de regresión lineal múltiple, que fue identificada por el $R^{2}$ lo que permitió medir la incidencia de la Variable Independiente sobre la Variable Dependiente. Finalmente se utilizó el anova con un análisis de varianza que permitió encontrar la significatividad de los factores estudiados.

\section{ANÁLISIS Y DISCUSIÓN DE RESULTADOS}

Tal como consta en la figura 1, las empresas evidencian un crecimiento en su capacidad instalada promedio del período, medida a través de la cuenta Activo Total. Las empresas presentan un grado de crecimiento, que oscila entre el $5 \%$ al $10 \%$ de aquellas que menos crecieron y en un rango del $21 \%$ al $28 \%$ las que más crecieron en activo total, es decir, en capacidad instalada. La figura 2 , exhibe la variación del crecimiento medida con el indicador de Producción, a través de la cuenta Costo de ventas. El nivel de variación del costo de ventas de las empresas estudiadas oscila entre $19 \%$ y el $27 \%$ de las empresas que menos crecieron y del $36 \%$ al $42 \%$ de las que mayor crecimiento presentaron como promedio del período. Al respecto la literatura revela que el proceso de adaptación a los cambios del entorno se ve reflejado en el aumento de la capacidad productiva de las empresas mediante la adquisición de nuevos recursos (Blásquez, et. al. 2006). Los resultados encontrados en el presente estudio, son coherentes con esta aseveración.

La figura 3 indica la variación del crecimiento empresarial medido con el indicador de Ventas a través de la cuenta Ingresos operacionales. Las empresas presentan un grado de crecimiento en ventas, que oscila entre el $18 \%$ al $23 \%$ para aquellas que menos crecieron y un $65 \%$ de la empresa que más creció. Es decir, el crecimiento empresarial implica "cambios abruptos" a decir de Muñoz-Salgado, Nava-Rogel y Rangel (2013) estos cambios que se hacen visibles al exterior como el aumento de las dimensiones de la empresa en infraestructura y participación en el mercado en el que compiten. 
Figura 1: Crecimiento empresarial medido con Activo Total

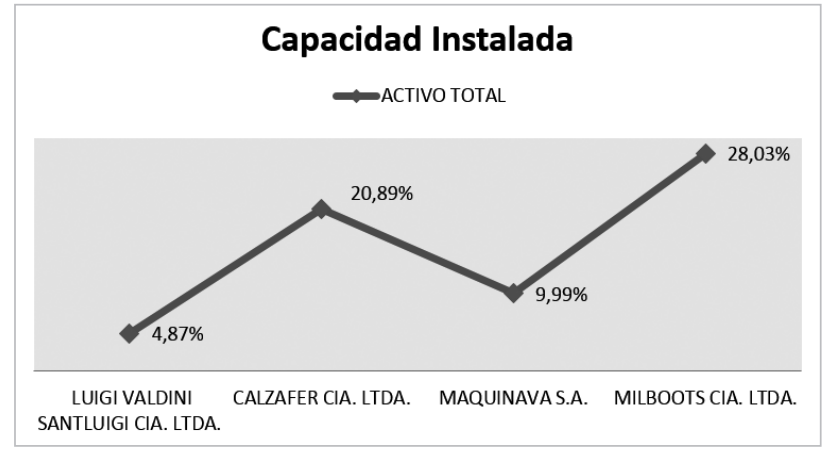

Elaboración propia

Fuente: Superintendencia de Compañías

Figura 2: Crecimiento empresarial medido con Costo de Ventas

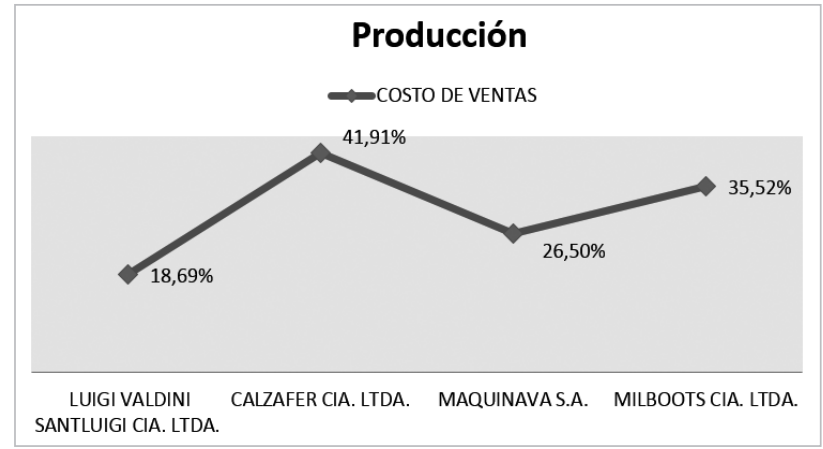

Elaboración propia

Fuente: Superintendencia de Compañías

Figura 3: Crecimiento empresarial medido con Ingresos Operacionales

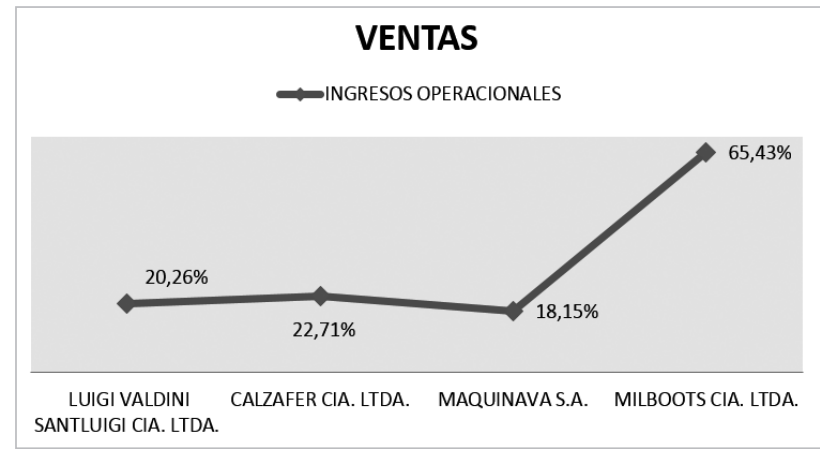

Elaboración propia

Fuente: Superintendencia de Compañías 
Tanto el crecimiento de la capacidad instalada como el crecimiento en producción y ventas de las empresas estudiadas, constituyen la evidencia del progreso de una organización que cumple sus objetivos utilizando y ajustando su capacidad interna a las demandas del entorno (Aguilera, 2010) y que deja entrever que se está gestando conocimiento (Guadamillas, 2006; Velásquez, et. al. 2010; García, y Romero, 2010) al interior de las empresas, producido por la experiencia adquirida a lo largo del tiempo.

A propósito de lo expuesto en el párrafo anterior, el análisis de la variable supervivencia muestra que la empresa de fabricación de calzado Luigi Valdini tiene 32 años en el mercado, mientras que las empresas Calzafer y Maquinava llevan un promedio de 15 años de funcionamiento, finalmente la empresa Milboots con 4 años en el mercado es la compañía más joven del grupo estudiado. Cabe destacar que a pesar de que la compañía Milboots lleva 4 años en el mercado, es la que evidencia un mayor y más rápidocrecimientoempresarial con una variación del indicador capacidad instalada de $28 \%$ promedio del periodo y un indicador de variación de ventas de 65\% promedio del periodo, siendo la variación más alta del grupo de empresas en todo el período. La revisión de la literatura permite identificar a la edad o el número de años que permanece en el mercado desde su iniciación, como uno de los factores determinantes para la supervivencia (Segovia y Camacho, 2012; Aguilera y Virgen, 2014), en un estudio empírico realizado y reflejado en la revisión de la literatura, se encontró que la edad de las empresas afecta de manera negativa en la probabilidad de fracaso empresarial (Baltar, et. al., 2011).

En una segunda etapa de análisis, se procede a la comprobación de la hipótesis ingresandotres indicadores para la variable crecimiento (véase tabla 1) y no se encontró relación de la variable Supervivencia con el Activo Total (cor: 0.3908) por lo que se la retiró del modelo estadístico.

\section{Tabla 2. Coeficientes estimados}

\begin{tabular}{|c|c|}
\hline $\begin{array}{c}\text { Coeficiente de } \\
\text { correlación }\end{array}$ & 0.99 \\
\hline $\mathrm{R}^{2}$ & 0.9998 \\
$\mathrm{R}^{2}$ ajustado & 0.9995 \\
\hline Anova & \\
Producción & 0.009338 \\
Ingresos & 0.018112 \\
\hline \multicolumn{2}{|c|}{ Signif. codes: $0^{(* * *)} 0.001^{(* * \prime} 0.01^{(*)}$} \\
\hline
\end{tabular}

Elaboración propia

Fuente: Explotación de los datos en el Software R Project

De modo que para la comprobación de la hipótesis planteada acerca de la incidencia de la Variable Independiente crecimiento empresarial sobre la Variable Dependiente supervivencia, el análisis de correlación que se obtiene refleja una correlación alta y positiva de 0.99 con Ingresos Operacionales (ventas) y Costo de Ventas (producción) respectivamente, tal como se observa en la tabla 2. Por otro lado, en la tabla 2 se presentan además los coeficientes de correlación, regresión lineal múltiple, anova y significatividad del modelo estadístico; al aplicar la regresión lineal múltiple, se encuentra que las Variables Costo de Ventas (producción) e Ingresos Operacionales (Ventas) inciden en los niveles de supervivencia con un $\mathrm{R}^{2}$ de 0.9998 y un $\mathrm{R}^{2}$ ajustado de 0.9995, finalmente, el anova en relación a la producción exhibe un $0.009338^{* *}$ y en relación a los ingresos un $0.018112^{*}$, los resultados cercanos a cero indican una alta significatividad del modelo lo que determina la validez del mismo para 
Figura 4: Análisis de regresión y correlación

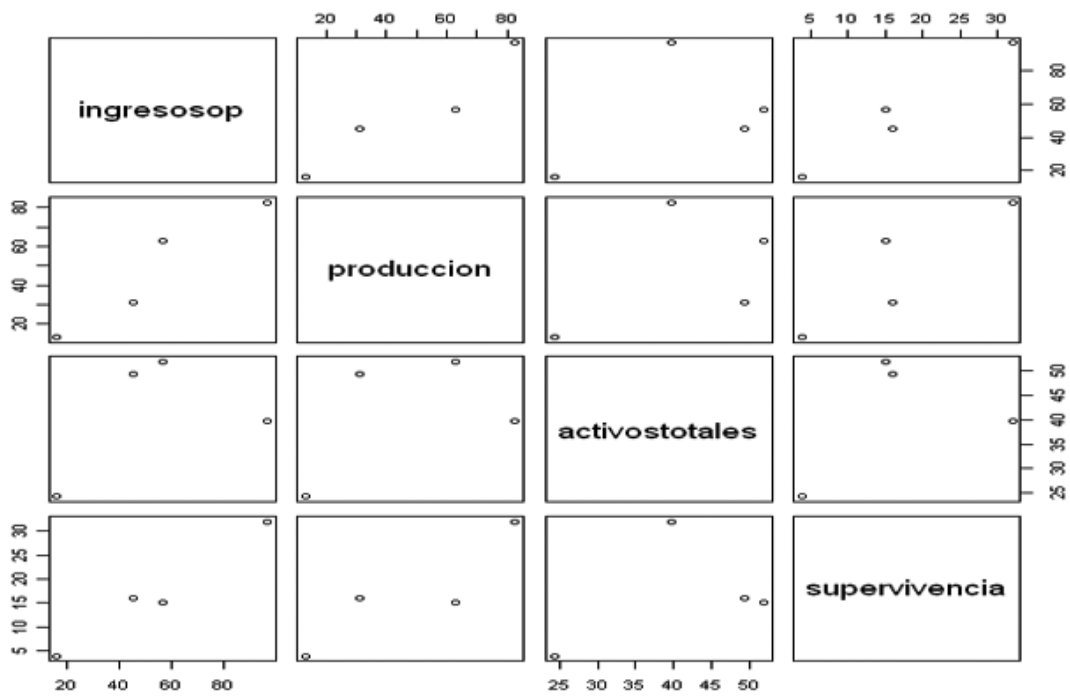

Elaboración propia.

Fuente: Superintendencia de Compañías

explicar el fenómeno de supervivencia, por lo que se puede evidenciar que la variable de mayor incidencia sobre la supervivencia de las empresas es producción (costo de ventas). En la figura 4 se presenta el plot generado por el software estadístico $R$ project que permite obtener una visión general del modelo.

Los resultados evidencian que cuando las empresas crecen en producción $\mathrm{y}$ ventas, aumenta la probabilidad de supervivencia, es decir, cuanto más grande es el tamaño de la organización será mayor la probabilidad de seguir en el mercado, por lo tanto, los resultados encontrados son coherentes con lo establecido en la literatura (Bordonaba, et. al., 2008; Segovia y Camacho, 2012) para el caso de las empresas del sector fabricación de calzado en Tungurahua-Ecuador.

\section{CONCLUSIONES}

$\mathrm{Si}$ bien los resultados presentados coinciden con lo expresado en la literatura en otros contextos y realidades, cabe destacar también que las características del sector (Melgarejo, et. al., 2007; Simón, 2012) en este caso de fabricación de calzado, son importantes para el análisis de la supervivencia. En la provincia de Tungurahua, existe una aglomeración de empresas, conformada no solo por empresas establecidas como las estudiadas, sino también por empresas de nueva creación que generan empleo y es necesario asegurar la estabilidad laboral y la continuidad de estas empresas a través de una certera estrategia de crecimiento, potenciando la productividad de los trabajadores en el marco del objetivo empresarial (Melgarejo, et. al., 2007). 
Una de las más importantes conclusiones del presente estudio, evidencia que las empresas que cuentan con un aumento del volumen de producción y por lo tanto de ventas (asumiendo que el aumento de producción es una reacción racional de las empresas a la demanda del mercado), son precisamente las que llevan más tiempo en el mercado, es decir, las que presentan mayor posibilidad de supervivencia, representada por su tamaño y edad.

\section{RECOMENDACIONES Y FUTURAS INVESTIGACIONES}

Para el presente estudio, no se consideraron algunos factores e indicadores de crecimiento como las estrategias de internacionalización, la responsabilidad social, el perfil del empresario emprendedor, la estructura y sucesión de la empresa, ni la inversión en $\mathrm{I}+\mathrm{D}$, reflejados en la revisión de la literatura, lo cual promueve futuros estudios que deberían abarcar estos aspectos, en la misma línea de investigación. Por otro lado, la metodología utilizada en este estudio podría ser aplicada en otros sectores económicos de la región lo cual permitirá hacer análisis comparativos y finalmente, un estudio de caso de la empresa Milboots, que es el referente de crecimiento rápido en el presente estudio, podría propiciar un trabajo de investigación futuro para establecer los factores de crecimiento en un caso de éxito en la provincia de Tungurahua.

En base a los resultados y a la comprobación de la hipótesis respecto de la relación entre el crecimiento y la supervivencia de las empresas estudiadas, se considera de primordial importancia proponer a las empresas más pequeñas del sector de fabricación de calzado la opción estratégica de crecer, no solamente en volumen o dimensión, sino a través de acuerdos de cooperación/alianzas estratégicas, con otras empresas del mismo sector. La estrategia de crecimiento en base a cooperación guarda coherencia con la idea de asociatividad que promueven actualmente el gobierno nacional y el gobierno provincial de Tungurahua.

\section{REFERENCIAS BIBLIOGRÁFICAS}

Aguilera, A. (2010). Direccionamiento estratégico y crecimiento empresarial: algunas reflexiones en torno a su relación. Pensamiento y Gestión from Business Economics and Theory Collection [revista en Internet] [Acceso 7 de febrero de 2017]; 28:85-106. Disponible en: http://rcientificas. uninorte.edu.co/index.php/pensamiento/article/viewFile/1020/641

Aguilera, A. y Puerto, D. (2012). Crecimiento empresarial basado en la Responsabilidad Social. Pensamiento \& Gestión 32. [revista en Internet] [Acceso 7 de febrero de 2017]; 32:1-26. Disponible en: http://www.redalyc.org/articulo.oa?id=64623932002

Aguilera, A. y Virgen, V. (2014). Principales indicadores de crecimiento empresarial en las pequeñas y medianas empresas: caso Santiago de Cali - Colombia. Revista Internacional Administración \& Finanzas, [revista en Internet] [Acceso 7 de febrero de 2017]; 7(6):27-43. Disponible en: https:// papers.ssrn.com/sol3/papers.cfm?abstract_id=2334385

Arias, A. y Quiroga, R. (2008). Cese de actividades de las pymes en el área metropolitana de Cali (2000-2004): un análisis de supervivencia empresarial. Cuadernos de Administración, [revista en Internet] [Acceso 7 de febrero de 2017]; 21(35):249-277. Disponible en: http://www.scielo.org. co/scielo.php?script=sci_abstract\&pid=S0120-35922008000100011 
Baltar, E. Fernández, S. Vivel, M. y Neira, I. (2011). Supervivencia das empresas innovadoras españolas: efectos da innovación. Revista Galega de Economía, [revista en Internet] [Acceso 7 de febrero de 2017]; 21(2):107-132. Disponible en: http://www.redalyc.org/pdf/391/39124539005.pdf

Blázquez, F. Dorta, J. y Verona, M. (2006). Concepto, perspectivas y medida del crecimiento empresarial. Cuadernos de Administración. [revista en Internet] [Acceso 7 de febrero de 2017]; 19(31):165195. Disponible en: http://www.redalyc.org/pdf/205/20503107.pdf

Bordonaba, M. Lucia, L. y Polo, Y. (2008). Análisis de la salida del mercado. Fracaso vs. Abandono en la franquicia. Cuadernos de Economía y Dirección de la Empresa. [revista en Internet] [Acceso 7 de febrero de 2017]; 12(39):033-058. Disponible en: http://www.elsevier.es/es-revista-cuadernos-economia-direccion-empresa-cede-324-articulo-analisis-salida-del-mercado-fracaso-S1138575809700349

García, J. y Romero, M. (2010). Efectos de la inversión en I+D sobre el crecimiento empresarial. Revista Globalización Competitividad y Gobernabilidad. [revista en Internet] [Acceso 7 de febrero de 2017]; 4(2):16-25. Disponible en: https://gcg.universia.net/article/view/381/efectos-inversion-i-d-crecimiento-empresarial-

Guadamillas, F. (2006). Conocimiento organizativo, innovación y crecimiento empresarial: el caso del Grupo Tecnobit. Universia business review-actualidad económica [revista en Internet] [Acceso 7 de febrero de 2017]; 12: 50-65. Disponible en: http://www.redalyc.org/pdf/433/43301205.pdf

Instituto Nacional de Estadística y Censos (2010). Encuesta de Manufactura y Minería [base de datos en Internet]. Ecuador. Disponible en: http://www.inec.gob.ec/estadisticas/?option=com_content\&view=article\&id=68

Instituto Nacional de Estadística y Censos (2013) [base de datos en Internet]. Ecuador. Disponible en: http://www.ecuadorencifras.gob.ec/

Melgarejo, Z. Arcelus, F. y Simon, K. (2007). Una evaluación crítica del potencial de supervivencia de las sociedades laborales [revista en Internet] [Acceso 7 de febrero de 2017]; 59:181-202. Disponible en: http://www.ciriec-revistaeconomia.es/banco/5907_Melgarejo_et_al.pdf

Ministerio de Industrias y Productividad (2014). Ecuador. Disponible en: http://www.industrias.gob.ec/

Muñoz-Salgado, F. Nava-Rogel, R. y Rangel Magdaleno, J. (2013). Capacidades innovadoras como estrategia de crecimiento en PYMES familiares. Revista de Empresa Familiar [revista en Internet] [Acceso 7 de febrero de 2017]; 3(2), 29-41. Disponible en: https://dialnet.unirioja.es/servlet/articulo?codigo $=4627592$

Plan Nacional del Buen Vivir (2013 - 2017). Ecuador. Disponible en: http://www.planificacion.gob.ec/

Puerto, D. (2010). La globalización y el crecimiento empresarial a través de estrategias de internacionalización. Pensamiento y Gestión from Business Economics and Theory Collection. [revista en Internet] [Acceso 7 de febrero de 2017]; 28: 171-195. Disponible en: http://www.scielo.org.co/pdf/ pege/n28/n28a09.pdf

Segovia, M. y Camacho, M. (2012). ¿Qué indicadores económicos financieros podrían condicionar la decisión del experto independiente sobre la supervivencia de una empresa en su fase pre concursal? evidencia empírica en España. Cuadernos de Contabilidad. [revista en Internet] [Acceso 7 de febrero de 2017]; 13(32): 97-119 Disponible en: http://cuadernosdecontabilidad.javeriana.edu. co/vol13_n_32/vol13_32_4.pdf 
Simón, V. Revuelto, L. Medina, A. (2012). La influencia de la formación, la experiencia y la motivación para emprender en la supervivencia de las empresas de nueva creación. Estudios gerenciales, [revista en Internet] [Acceso 7 de febrero de 2017]; 28:237-262. Disponible en: https://www.icesi. edu.co/revistas/index.php/estudios_gerenciales/article/view/1487/html

Superintendencia de Compañías (2013-2017) [base de datos en Internet]. Ecuador. Disponible en: http://www.supercias.gob.ec/portal/

Velásquez, F. Quintero, A. Sánchez, F. y Burgos, S. (2010). Grupo corporativo eficacia, una organización que crece y hace crecer: momentos de cambio. Estudios Gerenciales from Business Economics and Theory Collection. [revista en Internet] [Acceso 7 de febrero de 2017]; 26(115):181-200. Disponible en: https://www.icesi.edu.co/revistas/index.php/estudios_gerenciales/article/view/365/365

\section{Para citaciones:}

Morales-Carrasco, L. V., Morales-Ramos, K. E., \& Valle-Alvarez, A. T. (2017). Crecimiento y supervivencia empresarial en el sector de fabricación de calzado en Ecuador. Panorama Económico, 25, 1, pp. 43-56.

\section{AUTORES}

Lilián Victoria Morales-Carrasco

Profesora e Investigadora de la Universidad Tecnica de Ambato en Ecuador.

\section{Karla Estefanía Morales-Ramos}

Profesora e Investigadora de la Universidad Tecnica de Ambato en Ecuador. 